\title{
Eukaryotic Origins: How and When Was the Mitochondrion Acquired?
}

\author{
Anthony M. Poole ${ }^{1,2,3}$ and Simonetta Gribaldo ${ }^{4}$ \\ ${ }^{1}$ School of Biological Sciences, University of Canterbury, Christchurch 8140, New Zealand \\ ${ }^{2}$ Biomolecular Interaction Centre, University of Canterbury, Christchurch 8140, New Zealand \\ ${ }^{3}$ Allan Wilson Centre for Molecular Ecology and Evolution, University of Canterbury, Christchurch \\ 8140, New Zealand \\ ${ }^{4}$ Institut Pasteur, Unité Biologie Moléculaire du Gene chez les Extrêmophiles, Département \\ de Microbiologie, Paris 75724, France \\ Correspondence: anthony.poole@canterbury.ac.nz; simonetta.gribaldo@pasteur.fr
}

Comparative genomics has revealed that the last eukaryotic common ancestor possessed the hallmark cellular architecture of modern eukaryotes. However, the remarkable success of such analyses has created a dilemma. If key eukaryotic features are ancestral to this group, then establishing the relative timing of their origins becomes difficult. In discussions of eukaryote origins, special significance has been placed on the timing of mitochondrial acquisition. In one view, mitochondrial acquisition was the trigger for eukaryogenesis. Others argue that development of phagocytosis was a prerequisite to acquisition. Results from comparative genomics and molecular phylogeny are often invoked to support one or the other scenario. We show here that the associations between specific cell biological models of eukaryogenesis and evolutionary genomic data are not as strong as many suppose. Disentangling these eliminates many of the arguments that polarize current debate.

\begin{abstract}
$A$ dvances across the biological sciences have Ain recent years shed considerable light on the evolution of the eukaryote cell. Reconstruction of the most recent ancestor of all modern eukaryotes (also called last eukaryotic common ancestor or LECA) visible through the lens of comparative genomics has led to a consensus view that, before the diversification of eukaryotes into the extant phyla we see today, the major features of the eukaryote cell had already evolved (Koonin 2010; Poole 2010; Koumandou et al. 2013). This is testimony to the power of
\end{abstract}

this methodology, yet at the same time, it reveals its Achilles' heel; what emerges is a LECA that is so completely eukaryotic in nature as to render it wholly unremarkable. Consequently, the path from the initial ancestor of the eukaryotic lineage (also called first eukaryotic common ancestor, or FECA) to the LECA is effectively an event horizon (Jékely 2005); although gene duplications can be traced across this horizon (Makarova et al. 2005), the defining features of the eukaryote cell all evolved before the diversification of modern eukaryotes. The real chal-

Editors: Patrick J. Keeling and Eugene V. Koonin

Additional Perspectives on The Origin and Evolution of Eukaryotes available at www.cshperspectives.org

Copyright (C) 2014 Cold Spring Harbor Laboratory Press; all rights reserved; doi: 10.1101/cshperspect.a015990

Cite this article as Cold Spring Harb Perspect Biol 2014;6:a015990 
lenge is, thus, to establish how and in what order these features arose in the lineage leading to the LECA.

Among hallmark eukaryote features, the timing of mitochondrial acquisition has received particular attention. There is unanimous agreement on three aspects of mitochondrial origins. First, it is beyond doubt that all known present-day eukaryotes descend from an ancestor that already possessed a mitochondrion (Müller et al. 2012). The Archezoa hypothesis, which proposed that a few amitochondriate lineages represent the earliest divergences before mitochondrial acquisition (Cavalier-Smith 1987), has been unequivocally falsified (Embley and Martin 2006; van der Giezen 2009). Whereas eukaryotes that are genuinely ancestrally amitochondriate could plausibly persist, this appears on current data to be unlikely. Second, there is full agreement that one of the partners in this association was a bacterial cell (Gray 2012). Finally, there is no doubt that the host cell is more closely related to modern archaea than it is to any lineage of bacteria, although its precise nature is a matter of ongoing debate (Embley and Martin 2006; Poole and Penny 2007b; Gribaldo et al. 2010; Koonin 2010; Guy and Ettema 2011; Forterre 2013; Martijn and Ettema 2013).

Endosymbiosis is a process that has had a profound impact on all extant eukaryotes, and is one of the clearest examples of an evolutionary transition (Maynard Smith and Szathmáry 1995). Mitochondrial acquisition is so fundamental that it is ubiquitously reflected in modern eukaryote cell biology; modern mitochondria, mitosomes, and hydrogenosomes all trace back to this ancestral endosymbiont (Muller et al. 2012). Given the huge energetic gains associated with oxidative phosphorylation (Lane 2011), it seems reasonable to expect that acquisition of mitochondria was so advantageous that mitochondrion-bearing lineages ultimately swept to fixation, explaining why no "living fossils" of intermediate stages persist. This makes it particularly difficult to establish when the mitochondrion was acquired along the path leading to the LECA. Given this uncertainty, the numerous scenarios for eukaryote origins reveal three recurring points of debate: the timing (i.e., whether mitochondrial acquisition was an early or a late step in eukaryogenesis), the nature of the host (a protoeukaryote or an archaeon), and the mechanism of acquisition (syntrophy, bacteriovory, or phagotrophy). Traditionally, these different features of models for eukaryote origins have been strongly coassociated (e.g., an early acquisition by an archaeal host via syntrophy [reviewed in Lopez-Garcia and Moreira 1999] or a late acquisition of mitochondria by a protoeukaryote host via phagotrophy [Cavalier-Smith 2002]). In this article, we argue that coassociation has led to a lightning rod effect (Sober 1999) in which refuting a "bad" idea leads to erroneous "refutation" of associated good ideas.

Phylogeny and comparative genomics analyses have resulted in enormous progress on eukaryote origins. It is now clear that all eukaryotes evolved from a mitochondrion-bearing ancestor (Müller et al. 2012), that the main features of modern eukaryote cell architecture were present in that ancestor (Koumandou et al. 2013), and that there has been extensive transfer of genes of mitochondrial origin into the eukaryote nuclear genome (Esser et al. 2004). However, such results do not obviously fit only one scenario. We therefore examine whether phylogenomic analyses are sufficiently specific as to be compatible with only one scenario, and whether timing, host type, and mechanism of acquisition are obligatorily coassociated. We find that existing tests are not sufficiently specific to distinguish between scenarios, and key features of traditional models are not obligatorily coassociated. Further progress is possible, but rests on being clear on what these analyses can and cannot tell us. Indeed, several recent models are starting to pull apart these historical coassociations and we argue that this trend serves to highlight points of consensus, which are far greater than may be expected given the, at times, vigorous nature of debate. We believe this will lead to a more nuanced view of eukaryote origins because specific assertions on timing, nature of the host, and mechanism of acquisition can, and should, be independently assessed. 


\section{THE INFERENCE OF A MITOCHONDRION IN THE LECA DOES NOT NECESSARILY IMPLY THAT MITOCHONDRIAL ACQUISITION COINCIDES WITH EUKARYOTE ORIGINS}

If the unequivocal presence of a mitochondrion in the LECA has been a fantastic accomplishment of the application of phylogenetic methods to eukaryotes, the rejection of the Archezoa hypothesis has caused significant upheaval, with not wholly justified consequences. Eschewing the view that a fully formed eukaryote cell was the host to the mitochondrial ancestor, numerous alternative models emerged or were reinvigorated (Martin et al. 2001) that place mitochondrial acquisition as a very early event in eukaryogenesis (i.e., closer to the FECA than the LECA) (Fig. 1). Although many have since fallen from favor, syntrophic models based on metabolic interactions between at least one bacterium and an archaeon (Martin and Müller 1998; Moreira and Lopez-Garcia 1998; LopezGarcia and Moreira 1999) remain popular. Recent phylogenetic analyses (Rivera and Lake 2004; Cox et al. 2008; Guy and Ettema 2011; Williams et al. 2012) showing eukaryotes emerging from within Archaea are commonly assumed as consistent with these models, and the view that eukaryogenesis was driven by mitochondrial acquisition has become associated with this phylogenetic topology (i.e., mitochondrion-first models) (Fig. 1), and have somehow led

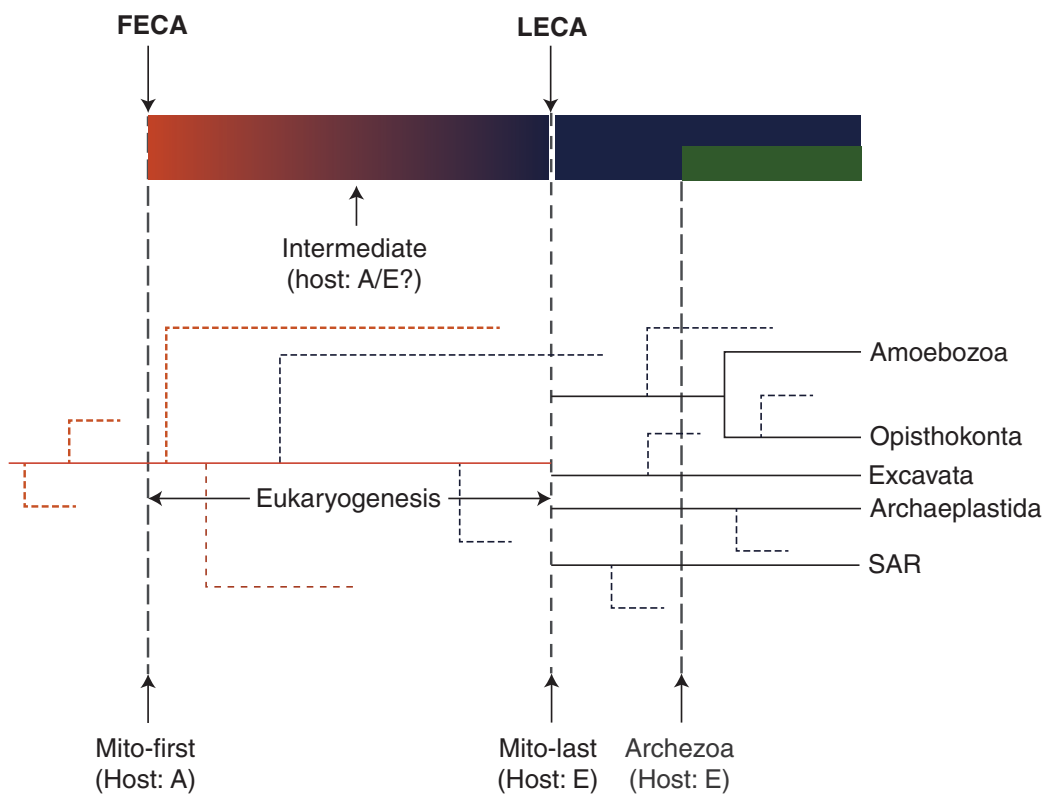

Figure 1. Timing of mitochondrial acquisition in eukaryogenesis. The relative timing of the acquisition of the mitochondrion during the process of eukaryogenesis from the FECA to the LECA (orange to blue shading) varies from scenarios in which this is the first step ("Mito-first") to those in which it is the last step ("Mito-last"). Note that there is a difference between the mitochondrion-last and Archezoa models. When the mitochondrion is the final step, the host is a fully formed eukaryotic cell. In the Archezoa model, acquisition was proposed to have occurred subsequent to early diversification of a few eukaryote lineages (depicted schematically by a blueto-green change). This model has been falsified so is in gray to reflect this. As discussed in the text, available data make it difficult to support either the Mito-first or Mito-last scenario. In particular, the absence of known premitochondrial lineages cannot be taken as an argument for Mito-first scenarios, as extinction of intermediate forms (dotted lines) is a natural process in evolution that has occurred before, during, and after eukaryogenesis. However, there may be considerable differences between "intermediate" scenarios, including with regard to the nature of the host (phylogenetically an archaeon or eukaryote). Major Eukaryotic groups are labeled and indicative relationships are presented. 
to the view that eukaryote origins require special explanation (Lane 2011).

We need to be wary of the lightning rod effect (Sober 1999) here, however. The inference of mitochondria in the LECA and consequent rejection of the Archezoa hypothesis do not necessarily reject the existence of some eukaryotic characters in the host before mitochondrial acquisition. Consequently, a tree topology in which eukaryotes emerge from Archaea does not show that eukaryotic origins coincide with mitochondrial acquisition. Indeed, comparative genomics has shown that numerous eukaryotic features trace to the LECA. These are now becoming too extensive to list, but include meiosis (Ramesh et al. 2005; Egel and Penny 2008), the nuclear pore complex (Mans et al. 2004; Bapteste et al. 2005; Neumann et al. 2010) and endomembrane system (Jékely 2007b; Koumandou et al. 2013), the spliceosome and spliceosomal introns (Collins and Penny 2005; Roy and Irimia 2009), small nucleolar RNPs (Gardner et al. 2010; Hoeppner and Poole 2012), and linear chromosomes with telomeres (Nakamura and Cech 1998; Fulneckova et al. 2013). Consequently, all these features can be traced back to the same timepoint (the LECA), but establishing in what order these features evolved before LECA is nontrivial.

Importantly, all models for the origin of the eukaryote cell (including syntrophic ones) imply numerous intermediate stages; starting from some ancestral state, endomembranes, chromosome architecture, reproduction, and even processing of messenger RNAs have all been fundamentally changed. It should be obvious that the emergence of all these characters requires the existence of intermediate and now extinct lineages (Fig. 1), and there is no reason why the mitochondrion would represent an exception.

Indeed, to suggest no diversification of forms would require the rather special evolutionary case of a trunk containing enormous evolutionary changes without speciation (Lane 2011; although, see in-article reviewer comments and Lang 2014 for robust arguments against this position). We can think of no good reasons why descent with modification (Darwin 1859) would fail to generate diversity in the case of eukaryotic origins. Given that the partial list above deals with molecular features that do not leave fossils, it may simply be a case of extinction of intermediate forms (Poole and Penny 2007b; Poole 2010), as has been clearly shown for lineages and features more amenable to fossilization (Donoghue and Purnell 2005).

A selective sweep may be hard for many to accept because it seems difficult to understand why lineages that have never possessed mitochondria would not persist when some modern lineages have "regressed" and lost mitochondrial function. Here, we must be careful to recognize that secondary losses of mitochondria are only partial. Remnants of mitochondria and their genes likely persist in amitochodriate lineages (Gabaldon and Huynen 2003; Gabaldon and Huynen 2007; Müller et al. 2012). A competition experiment between a secondarily amitochondriate eukaryote and a hypothetical premitochondrial eukaryote is necessary to resolve this debate, but is impossible if the latter no longer exists.

To conclude, although the acquisition of mitochondria was obviously an important event in the path to the LECA, the fact that no presentday eukaryotes derive from an ancestrally amitochondriate lineage cannot be used to argue that the first stages of eukaryogenesis coincide with mitochondrial acquisition; this observation is agnostic to the timing of mitochondrial origins.

\section{MITOCHONDRIAL SYMBIOSIS DID NOT OCCUR IMMEDIATELY BEFORE THE LECA}

The absence of premitochondrial lineages and apparent radiation at the base of the eukaryotic tree may support the idea that the process that led to establishment of mitochondrial symbiosis was a rapid event that initiated shortly before the LECA (i.e., mitochondria-last scenarios) (Fig. 1) and possibly at the origin of a fast diversification of the major phyla we see today. In this case, the host would have already possessed well-developed eukaryotic characteristics. Again, we need to be wary of the lightning-rod effect here; mitochondria-last scenarios are distinct from the Archezoa hypothesis (Fig. 1). The 
Archezoa hypothesis placed mitochondrial acquisition post-LECA, and is firmly rejected. In contrast, mitochondria-late hypotheses place mitochondrial acquisition late in eukaryogenesis, but pre-LECA. Crucially, rejection of mitochondria-last scenarios is possible, but this is based on "different data" than those that led to rejection of the Archezoa hypothesis.

Comparative genomics of mitochondrial processes and structures show that the process that led to establishment of mitochondrial symbiosis is unlikely to have begun shortly before the LECA, as it required a substantial number of coevolutionary events both in the endosymbiont and its host, notably the evolution of ATP / ADP translocases (Amiri et al. 2003) and mitochondrial protein-import machinery (Dolezal et al. 2006; Tong et al. 2011). Moreover, if the process leading to mitochondrial endosymbiosis occurred right before the diversification of present-day eukaryotes, it would be expected that the nature of mitochondrial systems of bacterial origin inferred in the LECA would be very similar to those of the bacterial ancestor. However, this is not the case. For example, the ribosome of the mitochondrion in the LECA already had at least 19 novel eukaryotic-specific proteins compared to the original $\alpha$-proteobacterial ribosome (Desmond et al. 2011). These proteins were therefore added to the 54 initial proteins during establishment of endosymbiosis and before eukaryote diversification.

Other data are appearing that are deepening our understanding of the nature of host cell-mitochondrial endosymbiont coevolution, pre-LECA. For instance, the evolution of the endoplasmic reticulum-mitochondria encounter structure (ERMES), which links mitochondria to the endoplasmic reticulum via four proteins (Mmm1, Mmm2, Mdm10, Mdm12), was recently investigated (Wideman et al. 2013). The ERMES plays multiple roles spanning attachment, molecular transport of proteins and lipids, division, mitochondrial inheritance, and maintaining morphology (Wideman et al. 2013). On available data, ERMES is either a gain in the lineages leading to Excavata, Amoebozoa, and Opisthokonta, or could have been present in LECA, depending on the position of the root of the eukaryote tree (under the tree in Fig. 1, it is a feature of LECA). This work, therefore, only refutes mitochondria-last scenarios under certain rootings of the eukaryote tree. However, it further highlights the value in studying cellular machineries that have arisen as a result of coevolution of host cell and endosymbiont during the process that led to full mitochondrial establishment.

\section{DIFFERENT MODELS FOR EUKARYOTIC ORIGINS ARE COMPATIBLE WITH BOTH MITOCHONDRIA-EARLY AND MITOCHONDRIA-LATE SCENARIOS}

Although the exact nature of the symbiotic interaction between mitochondrial ancestor and host remains uncertain, phylogenetic analyses have clearly shown that the diversity of modern biochemistries seen in mitochondria, hydrogenosomes, and mitosomes have emerged through specialization and loss from a bacterial ancestor (van der Giezen and Tovar 2005; Embley 2006) with biochemical capabilities no more diverse than those found in free-living bacteria (Müller et al. 2012). There is full agreement that the mitochondrial ancestor was a bacterial cell of $\alpha$-proteobacterial origin (although identifying its closest extant relatives is still subject to uncertainty) (Esser et al. 2004; Fitzpatrick et al. 2006; Brindefalk et al. 2011; Thrash et al. 2011; Gray 2012; Rodriguez-Ezpeleta and Embley 2012; Viklund et al. 2012). There is, likewise, no doubt that the host was more closely related to modern archaea than it is to any lineage of bacteria (Embley and Martin 2006; Poole and Penny 2007b; Cox et al. 2008; Koonin 2010; Guy and Ettema 2011; Williams et al. 2012). Whether that means the host was an archaeon in cell biological terms is less certain.

As opposed to a sister relationship between Eukaryotes and Archaea indicated by trees rooted with ancient paralogs (also called three primary Domains or 3D scenarios) (Gogarten et al. 1989; Iwabe et al. 1989; Woese et al. 1990), several recent phylogenetic analyses of universally distributed proteins indicate an emergence of Eukaryotes from within the radiation of present-day archaea (also called two primary Do- 
A.M. Poole and S. Gribaldo

mains or 2D scenarios) (Cox et al. 2008; Guy and Ettema 2011; Williams et al. 2012; Lake and Sinsheimer 2013). Another class of results has emerged in phylogenomics, whereby similarities between eukaryote and archaeal gene sets are invoked as evidence that eukaryotes have an archaeal ancestry (Alvarez-Ponce et al. 2013). These results are frequently endorsed to support mitochondria-first models of eukaryogenesis involving an archaeal host and a bacterium (Fig. 1), yet without the necessary discriminatory power of phylogenetic analysis. The trouble with these types of analyses is that they are equally consistent with a sister relationship between Archaea and Eukaryotes in which the "archaeal component" of eukaryotic genomes was inherited from an ancestor shared with Archaea, not a modern archaeon. Indeed, current hypotheses on the evolutionary relationships between Archaea and Eukaryotes rely on limited phylogenetic data and surely deserve further analysis (Gribaldo et al. 2010).

Most importantly, and contrary to what is sometimes assumed, 3D scenarios are not ob- ligatorily mitochondria-last and 2D scenarios need not be mitochondria first (Fig. 2). In fact, the exact nature of the evolutionary relationships between Archaea and Eukaryotes does not provide much information on the timing of mitochondrial endosymbiosis. In both 2D and 3D trees, this event occurred before diversification of modern eukaryotes, so this may have happened early or late irrespective of the nature of the host (i.e., sister to or nested within the archaeal radiation) (Fig. 2). Notably, the cellular nature of the host that took up the mitochondrion is left unresolved even in the case of an archaeal origin of eukaryotes, as this may have been an archaeon in the case of an early acquisition, but also a lineage with some eukaryotic characteristics in the case of an intermediate or late acquisition (and, in this case, nothing distinguishes it from a classical premitochondrial eukaryotic lineage) (Fig. 1).

Likewise, it is important to note that not all 2D models proposing the origin of eukaryotes from the merging of bacteria and archaea imply mitochondrial endosymbiosis as the first
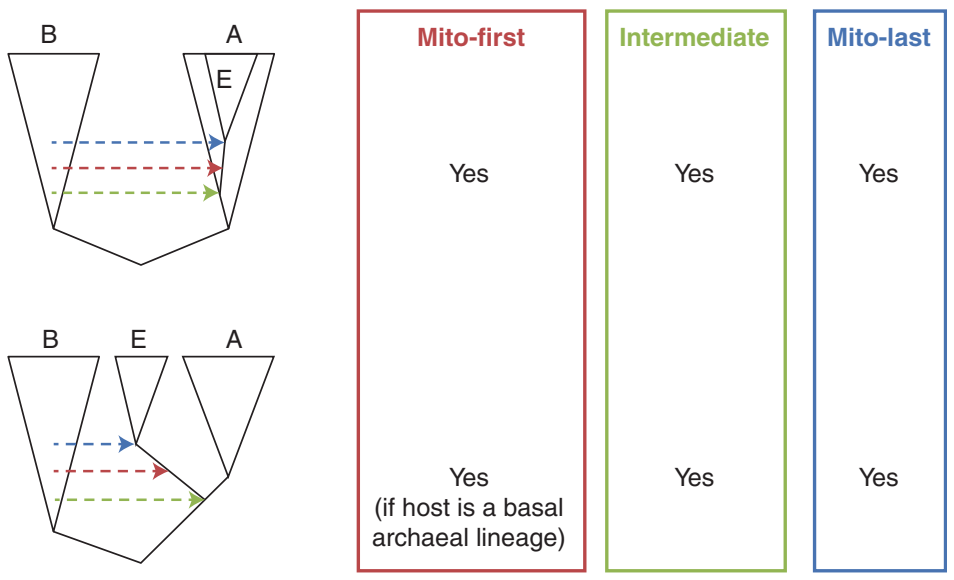

Figure 2. Alternative models for eukaryotic origins and different timing of mitochondrial acquisition are not incompatible. Contrary to what may be assumed, 2D hypotheses for eukaryotic origins (upper tree) do not necessarily imply mitochondria-first models and 3D models (lower tree) do not necessarily imply mitochondria-last models. Note that both $2 \mathrm{D}$ and 3D models require a stem from the FECA to the LECA along which eukaryogenesis occurred. If mitochondrial acquisition is the first step in eukaryogenesis, the host is, by definition, an archaeon. This is compatible with eukaryotes emerging from within archaea (2D), but also with 3D scenarios if they derive from a basal archaeal lineage. The mitochondrion-last model is agnostic as to the evolutionary relationship between eukaryotes and archaea because it only makes the statement that the final step in eukaryogenesis is mitochondrial acquisition, and the host can classically derive from the eukaryotic lineage (3D), but also from an archaeal lineage (2D) that would have developed eukaryotic features. 
step of eukaryogenesis (i.e., mitochondriafirst). For example, the synthrophy hypothesis of Moreira and Lopez-Garcia (1998) initially envisages a consortium composed of methanogens and $\delta$-proteobacteria in which the ancestor of mitochondria would become engaged subsequently.

\section{HOW WERE MITOCHONDRIA ACQUIRED?}

The mechanism of entry of the mitochondrial ancestor into its host is central to eukaryogenesis, yet this is often discussed in very limited terms despite the fact that endosymbiosis specifically requires one cell to take up stable residence in another cell. For endosymbiosis to emerge, there first needs to be interaction between host and endosymbiont. Three processes have been recognized as having the potential to permit this. These are bacteriovory (meaning, here, the specific case of one bacterium infiltrating another), syntrophy (in which two organisms interact biochemically without endosymbiosis), and phagocytosis (in which endosymbiont entry is via the phagocytic machinery). What distinguishes models for eukaryogenesis then is not so much the timing as the mechanism by which endosymbiosis is established.

Three key criteria are required to support cellular interactions leading to eukaryote cell origins (specifically between a bacterium and a host of eukaryotic/archaeal nature). These criteria are a cross-domain interaction, coevolutionary interaction, and cross-domain endosymbiosis (Table 1). Using these criteria, we consider each proposed mechanism in turn.

Syntrophy models are very well developed on biochemical grounds (Martin and Müller 1998; Moreira and Lopez-Garcia 1998; LopezGarcia and Moreira 1999), and clearly show two of these three criteria (Table 1); cross-domain interactions are documented between archaea and bacteria and there is evidence of a coevolutionary aspect to this interaction, for instance, the membrane bending observed in some syntrophic interactions. However, syntrophic interactions between partners stop there. No contemporary examples of a bacterial endosymbiont resident in an archaeal host are known. This indicates that the documented close association is sufficient for the interaction without internalization of the bacterial cell. If this were not the case, bacterial endosymbionts of Archaea would be as abundant as they are in Eukaryotes. Likewise, in syntrophy models, a symbiotic association must be established (Martin and Müller 1998; Lopez-Garcia and Moreira 1999), and the question here is whether strengthening this association through endosymbiosis was only possible following evolutionary changes that enabled either host to engulf or symbiont to infiltrate the host cell. It is not clear that the cell biological processes culminating in modern phagocytosis could not have been part of this process (Koumandou et al. 2013).

Bacteriovory by bacterial predators is a fascinating phenomenon. Species such as Bdellovibrio bacteriovorans have been shown to infiltrate the periplasm of their Gram-negative hosts (Stolp and Starr 1963; Martin 2002), and some species may even penetrate the cytoplasm (Guerrero et al. 1986). This shows that bacterial cells can gain entry to other bacterial cells, thereby indicating that intracellular access is not restricted to hosts with modern eukaryote cell architecture (Table 1; Fig. 3). In the case of bacterial infiltration (sensu Bdellovibrio), endosymbiosis can only be understood in terms of a failed predatory event. This best fits a coevolutionary model (Table 1) in which the host

Table 1. Three criteria must be met for a specific mechanism to be able to explain mitochondrial acquisition and endosymbiosis

\begin{tabular}{lccc}
\hline & Syntrophy & Bacteriovory & Phagotrophy \\
\hline Cross-domain interaction? & Yes & No & Yes \\
Contemporary examples of cross-domain endosymbiosis? & No & No & Yes \\
Evidence of coevolution? & Yes & Yes & Yes \\
\hline
\end{tabular}




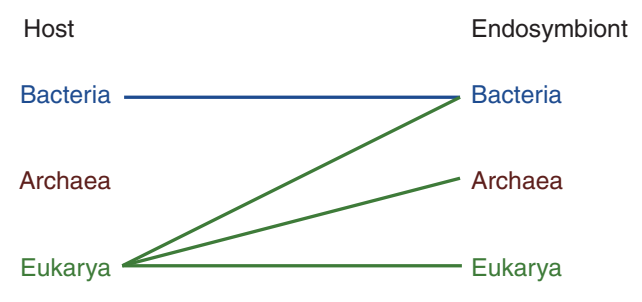

Figure 3. Known endosymbiosis across Bacteria, Archaea, and Eukaryotes. Bacterial endosymbionts of other bacteria are well known, the most striking examples being that of $M$. endobia, a $\gamma$-proteobacterial endosymbiont of $T$. princeps, a $\beta$-proteobacterium that is, in turn, an endosymbiont of mealybugs (von Dohlen et al. 2001; Husnik et al. 2013), and Bdellovibrio bacteriovorus, which infiltrates prey bacteria as part of its life cycle (Rendulic et al. 2004). Eukaryotes are known to house bacterial, archaeal, and eukaryotic endosymbionts. For instance, ciliates in microoxic, sulfidic environments carry both archaeal and bacterial endosybionts (Edgcomb et al. 2011), and the persistence of nucleomorphs in cryptophytes and chlorarachniophytes show these lineages independently acquired photosynthesis through engulfment of eukaryote algae (Curtis et al. 2012). No instances of Archaea-carrying endosymbionts have been documented. This is a requirement for models of eukaryote origins in which the host is cell-biologically archaeal. Note that this is different from a phylogenetic origin of eukaryotes within Archaea in which phagocytosis predates mitochondrial acquisition (Jékely 2007a; Poole and Neumann 2011; Martijn and Ettema 2013).

is infiltrated by the parasite, but instead of the parasite destroying its host, the parasite persists in the host and the host also survives. From such interactions, a stable endosymbiosis may emerge. Hence, bacteriovory may mechanistically explain stable bacterial-bacterial endosymbioses such as the case of Moranella endobia, a $\gamma$-proteobacterial endosymbiont of Tremblaya princeps, a $\beta$-proteobacterial endosymbiont of mealybugs (von Dohlen et al. 2001; Husnik et al. 2013). Likewise, Midichloria mitochondrii, an $\alpha$-proteobacterial endosymbiont of tick mitochondria (Sassera et al. 2006; Montagna et al. 2013), could be accounted for by this mechanism. However, although the case has been made for bacteriovory at the origin of the eukaryote cell (Davidov and Jurkevitch 2009), this mechanism fails to explain eukary- ote origins for the simple reason that there are no documented instances of a bacterial predator invading archaeal cells, so it does not fit the criterion of cross-domain interaction nor crossdomain endosymbiosis (Table 1). This absence of archaeal prey is notable not only for cytoplasmic or periplasmic cell entry, but also applies to other known mechanisms of bacteriovory such as wolf pack predation by Myxococcus and epibiontic attachment by Vampirococcus (Martin 2002).

The third process that could account for mitochondrial origins is phagotrophy. Comparative genomic studies of the molecular machinery for both phagocytosis (Yutin et al. 2009) and endocytosis (Dacks et al. 2008) reveal that components of each do trace back to the LECA (Koumandou et al. 2013). That some components are not universal requires further consideration, but we should bear in mind that post-LECA embellishments are a feature of most eukaryotes. For instance, there are clear examples of post-LECA gains in the evolution of specific versions of the nuclear pore complex, despite the fact a near-complete pore can be inferred in LECA (Neumann et al. 2010). What is more, phagocytosis has been insufficiently studied to ascertain the minimal machinery for this process. Add to that the observation that eukaryote genomes have undergone extensive protein family expansion via gene duplication both pre- and post-LECA (Makarova et al. 2005; Devos et al. 2006; Dacks et al. 2009; Lynch et al. 2011) and one finds there is nothing special about the observation that the phagocytic machinery has been embellished post-LECA.

As far as we can tell, resistance to involvement of phagotrophy in eukaryogenesis comes primarily from the mistaken belief that this mechanism is only compatible with the $3 \mathrm{D}$ topology-another instance of the lightningrod effect. However, the recent emergence of "phagocytosing archaeoan" models (Jékely 2007a; Yutin et al. 2009; Poole and Neumann 2011; Martijn and Ettema 2013) serves to illustrate that phagocytosis is not tied to a particular phylogenetic topology, even if it has been historically associated with the view that Eukaryotes and Archaea are sister lineages (Cavalier-Smith 
2002; Poole and Penny 2007a). In fact, models in which eukaryotes would emerge from an archaeal lineage having developed some mechanisms of phagocytosis are compatible with both 3D and 2D scenarios. Most importantly, these models are neutral on the relative timing of emergence of other eukaryotic features.

Embracing a phagotrophic archaeon as host for the mitochondrial ancestor allows us to sidestep the odd requirement of other models (endosymbiosis through syntrophy or predation between a bacterium and an archaeon), invoking a mechanism of acquisition for which there is no genuine precedent. Bacteriovory has only been reported between bacteria, and syntrophy has not been shown to lead to bacterial endosymbionts resident in archaea. A phagotrophic archaeon comfortably accounts for the ongoing and widespread cases of endosymbioses between eukaryote hosts and eukaryotic, bacterial, and archaeal endosymbionts (Fig. $3)$. As has been pointed out, all such endosymbioses are predicated on a biochemical association (Nowack and Melkonian 2010). Phagocytosis as a mechanism for engulfment or as a means of bacterial infiltration (Poole and Penny $2007 b$ ) is, in this regard, just a mechanism for entry and a means of establishing a wide variety of endosymbiont-host biochemical interactions (Nowack and Melkonian 2010).

Finally, it should be noted that nothing distinguishes a phagotrophic archaeon from a protoeukaryote in the pre-LECA era. Therefore, whether Eukaryotes are sister to Archaea or derive from them, the centrality of phagocytosis in eukaryote origins remains intact as a mechanism for endosymbiont entry (Cavalier-Smith 2002, 2009; Poole and Penny 2007b; Poole and Neumann 2011).

\section{CONCLUDING REMARKS}

Three aspects of eukaryote origins have been the subject of extensive debate: the timing of mitochondrial acquisition, nature of the host, and mechanism of acquisition. Although it is difficult to pin down the timing of mitochondrial acquisition, the field has made progress. It is now uncontroversial to argue that this occurred
pre-LECA and, as discussed here, that mitochondrial endosymbiosis was not the final event before diversification of modern eukaryotes.

Regarding the nature of the host, knowing the exact phylogenetic origins of eukaryotes is important, but it does not illuminate the set of cellular features that would have been present at the time of mitochondrial acquisition. Demonstrating a phylogenetic origin for eukaryotes within Archaea is helpful because it would imply that the host carried archaeal features. In this case, eukaryotes could be considered as a highly derived group of nucleate, mitochondrion-bearing Archaea. However, it does not follow that, in the path to modern eukaryotes, mitochondrial acquisition was obligatorily the first step in eukaryogenesis. The relative timing of the origins of modern eukaryote features traceable to LECA is hidden behind the LECA event horizon. If Archaea and Eukaryotes are related as per the three-domains tree, the picture is even murkier.

In examining the mechanism of mitochondrial acquisition, two conclusions are clear. First, phagocytosis is the sole mechanism that, on current data, can account for mitochondrial acquisition because it is the only process known that leads to cells of one domain living intracellularly in cells of another domain; eukaryotes play host to endosymbionts from all three domains. Bacteria are only known to play host to other bacteria and no archaea are known to carry endosymbionts of any kind. The common description of bacterial-bacterial endosymbioses as occurring between two prokaryotes is an instance in which preferential use of the term Prokaryote (Doolittle and Zhaxybayeva 2013) has clouded our understanding because it suggests bacterial-bacterial endosymbioses support hypothesized bacterial-archaeal endosymbioses when they do not. Second, given that basic phagocytic and endocytic machinery trace to the LECA, this provides a clear mechanism for mitochondrial acquisition. Models involving phagocytic archaea recognize that mitochondrial acquisition via phagocytosis is the best-supported mechanism, and illustrate that this can occur in a lineage that is phylogenetically archaeal, but on the path to eukaryogene- 
A.M. Poole and S. Gribaldo

sis. Regardless of whether eukaryotes are a derived group of archaea or sister to the archaea, the recognition that phagotrophy as a mechanism is not tied to one of these relationships is an important development.

\section{ACKNOWLEDGMENTS}

A.M.P. gratefully acknowledges receipt of a Royal Society of New Zealand Rutherford Discovery Fellowship. S.G. gratefully acknowledges support from the French Agence Nationale de la Recherche through the Investissement d'Avenir grant "Ancestrome" (ANR-10- BINF-01-01).

\section{REFERENCES}

Alvarez-Ponce D, Lopez P, Bapteste E, McInerney JO. 2013. Gene similarity networks provide tools for understanding eukaryote origins and evolution. Proc Natl Acad Sci 110: E1594-E1603.

Amiri H, Karlberg O, Andersson SG. 2003. Deep origin of plastid/parasite ATP/ADP translocases. J Mol Evol 56: 137-150.

Bapteste E, Charlebois RL, MacLeod D, Brochier C. 2005. The two tempos of nuclear pore complex evolution: Highly adapting proteins in an ancient frozen structure. Genome Biol 6: R85.

Brindefalk B, Ettema TJ, Viklund J, Thollesson M, Andersson SG. 2011. A phylometagenomic exploration of oceanic $\alpha$-proteobacteria reveals mitochondrial relatives unrelated to the SAR11 clade. PLoS ONE 6: e24457.

Cavalier-Smith T. 1987. The origin of cells: A symbiosis between genes, catalysts, and membranes. Cold Spring Harb Symp Quant Biol 52: 805-824.

Cavalier-Smith T. 2002. The phagotrophic origin of eukaryotes and phylogenetic classification of Protozoa. Int J Syst Evol Microbiol 52: 297-354.

Cavalier-Smith T. 2009. Predation and eukaryote cell origins: A coevolutionary perspective. Int J Biochem Cell Biol 41: 307-322.

Collins L, Penny D. 2005. Complex spliceosomal organization ancestral to extant eukaryotes. Mol Biol Evol 22: 1053-1066.

Cox CJ, Foster PG, Hirt RP, Harris SR, Embley TM. 2008. The archaebacterial origin of eukaryotes. Proc Natl Acad Sci 105: 20356-20361.

Curtis BA, Tanifuji G, Burki F, Gruber A, Irimia M, Maruyama S, Arias MC, Ball SG, Gile GH, Hirakawa Y, et al. 2012. Algal genomes reveal evolutionary mosaicism and the fate of nucleomorphs. Nature 492: 59-65.

Dacks JB, Poon PP, Field MC. 2008. Phylogeny of endocytic components yields insight into the process of nonendosymbiotic organelle evolution. Proc Natl Acad Sci 105: 588-593.
Dacks JB, Peden AA, Field MC. 2009. Evolution of specificity in the eukaryotic endomembrane system. Int J Biochem Cell Biol 41: 330-340.

Darwin C. 1859. On the origin of species by means of natural selection. J. Murray, London.

Davidov Y, Jurkevitch E. 2009. Predation between prokaryotes and the origin of eukaryotes. Bioessays 31: 748-757.

Desmond E, Brochier-Armanet C, Forterre P, Gribaldo S. 2011. On the last common ancestor and early evolution of eukaryotes: Reconstructing the history of mitochondrial ribosomes. Res Microbiol 162: 53-70.

Devos D, Dokudovskaya S, Williams R, Alber F, Eswar N, Chait BT, Rout MP, Sali A. 2006. Simple fold composition and modular architecture of the nuclear pore complex. Proc Natl Acad Sci 103: 2172-2177.

Dolezal P, Likic V, Tachezy J, Lithgow T. 2006. Evolution of the molecular machines for protein import into mitochondria. Science 313: 314-318.

Donoghue PC, Purnell MA. 2005. Genome duplication, extinction and vertebrate evolution. Trends Ecol Evol 20: 312-319.

Doolittle WF, Zhaxybayeva O. 2013. What is a prokaryote? In The prokaryotes (ed. Rosenberg E, DeLong EF, Lory S, Stackebrandt E, Thompson F), pp. 21-37. Springer, Berlin.

Edgcomb VP, Leadbetter ER, Bourland W, Beaudoin D, Bernhard JM. 2011. Structured multiple endosymbiosis of bacteria and archaea in a ciliate from marine sulfidic sediments: A survival mechanism in low oxygen, sulfidic sediments? Front Microbiol 2: 55.

Egel R, Penny D. 2008. On the origin of meiosis in eukaryotic evolution: Coevolution of meiosis and mitosis from feeble beginnings. Springer, Berlin.

Embley TM. 2006. Multiple secondary origins of the anaerobic lifestyle in eukaryotes. Philos Trans R Soc Lond B Biol Sci 361: 1055-1067.

Embley TM, Martin W. 2006. Eukaryotic evolution, changes and challenges. Nature 440: 623-630.

Esser C, Ahmadinejad N, Wiegand C, Rotte C, Sebastiani F, Gelius-Dietrich G, Henze K, Kretschmann E, Richly E, Leister D, et al. 2004. A genome phylogeny for mitochondria among $\alpha$-proteobacteria and a predominantly eubacterial ancestry of yeast nuclear genes. Mol Biol Evol 21: $1643-1660$.

Fitzpatrick DA, Creevey CJ, McInerney JO. 2006. Genome phylogenies indicate a meaningful $\alpha$-proteobacterial phylogeny and support a grouping of the mitochondria with the Rickettsiales. Mol Biol Evol 23: 74-85.

Forterre P. 2013. The common ancestor of archaea and eukarya was not an archaeon. Archaea 2013: 18.

Fulneckova J, Sevcikova T, Fajkus J, Lukesova A, Lukes M, Vlcek C, Lang BF, Kim E, Elias M, Sykorova E. 2013. A broad phylogenetic survey unveils the diversity and evolution of telomeres in eukaryotes. Genome Biol Evol 5: $468-483$.

Gabaldon T, Huynen MA. 2003. Reconstruction of the proto-mitochondrial metabolism. Science 301: 609.

Gabaldon T, Huynen MA. 2007. From endosymbiont to host-controlled organelle: The hijacking of mitochondrial protein synthesis and metabolism. PLoS Comput Biol 3: e219. 
Gardner PP, Bateman A, Poole AM. 2010. SnoPatrol: How many snoRNA genes are there? J Biol 9: 4.

Gogarten JP, Kibak H, Dittrich P, Taiz L, Bowman EJ, Bowman BJ, Manolson MF, Poole RJ, Date T, Oshima T, et al. 1989. Evolution of the vacuolar $\mathrm{H}^{+}$-ATPase: Implications for the origin of eukaryotes. Proc Natl Acad Sci 86: 66616665.

Gray MW. 2012. Mitochondrial evolution. Cold Spring Harb Perspect Biol 4: a011403.

Gribaldo S, Poole AM, Daubin V, Forterre P, Brochier-Armanet C. 2010. The origin of eukaryotes and their relationship with the Archaea: Are we at a phylogenomic impasse? Nat Rev Microbiol 8: 743-752.

Guerrero R, Pedros-Alio C, Esteve I, Mas J, Chase D, Margulis L. 1986. Predatory prokaryotes: Predation and primary consumption evolved in bacteria. Proc Natl Acad Sci 83: $2138-2142$.

Guy L, Ettema TJ. 2011. The archaeal “TACK” superphylum and the origin of eukaryotes. Trends Microbiol 19: 580 587.

Hoeppner MP, Poole AM. 2012. Comparative genomics of eukaryotic small nucleolar RNAs reveals deep evolutionary ancestry amidst ongoing intragenomic mobility. BMC Evol Biol 12: 183.

Husnik F, Nikoh N, Koga R, Ross L, Duncan RP, Fujie M, Tanaka M, Satoh N, Bachtrog D, Wilson AC, et al. 2013. Horizontal gene transfer from diverse bacteria to an insect genome enables a tripartite nested mealybug symbiosis. Cell 153: 1567-1578.

Iwabe N, Kuma K, Hasegawa M, Osawa S, Miyata T. 1989. Evolutionary relationship of archaebacteria, eubacteria, and eukaryotes inferred from phylogenetic trees of duplicated genes. Proc Natl Acad Sci 86: 9355-9359.

Jékely G. 2005. Glimpsing over the event horizon: Evolution of nuclear pores and envelope. Cell Cycle 4: 297-299.

Jékely G. 2007a. Origin of phagotrophic eukaryotes as social cheaters in microbial biofilms. Biol Direct 2: 3 .

Jékely G. 2007b. Origin of eukaryotic endomembranes: A critical evaluation of different model scenarios. $A d v$ Exp Med Biol 607: 38-51.

Koonin EV. 2010. The origin and early evolution of eukaryotes in the light of phylogenomics. Genome Biol 11: 209.

Koumandou VL, Wickstead B, Ginger ML, van der Giezen M, Dacks JB, Field MC. 2013. Molecular paleontology and complexity in the last eukaryotic common ancestor. Crit Rev Biochem Mol Biol 48: 373-396.

Lake JA, Sinsheimer JS. 2013. The deep roots of the rings of life. Genome Biol Evol 5: 2440-2448.

Lane N. 2011. Energetics and genetics across the prokaryoteeukaryote divide. Biol Direct 6: 35 .

Lang BF. 2014. Mitochondria and the origin of eukaryotes. In Endosymbiosis (ed. Löffelhardt W), pp. 3-18. Springer, Berlin.

Lopez-Garcia P, Moreira D. 1999. Metabolic symbiosis at the origin of eukaryotes. Trends Biochem Sci 24: 88-93.

Lynch M, Bobay LM, Catania F, Gout JF, Rho M. 2011. The repatterning of eukaryotic genomes by random genetic drift. Annu Rev Genomics Hum Genet 12: 347-366.

Makarova KS, Wolf YI, Mekhedov SL, Mirkin BG, Koonin EV. 2005. Ancestral paralogs and pseudoparalogs and their role in the emergence of the eukaryotic cell. Nucleic Acids Res 33: 4626-4638.

Mans BJ, Anantharaman V, Aravind L, Koonin EV. 2004. Comparative genomics, evolution and origins of the nuclear envelope and nuclear pore complex. Cell Cycle 3: 1612-1637.

Martijn J, Ettema TJ. 2013. From archaeon to eukaryote: The evolutionary dark ages of the eukaryotic cell. Biochem Soc Trans 41: 451-457.

Martin MO. 2002. Predatory prokaryotes: An emerging research opportunity. J Mol Microbiol Biotechnol 4: 467477.

Martin W, Müller M. 1998. The hydrogen hypothesis for the first eukaryote. Nature 392: 37-41.

Martin W, Hoffmeister M, Rotte C, Henze K. 2001. An overview of endosymbiotic models for the origins of eukaryotes, their ATP-producing organelles (mitochondria and hydrogenosomes), and their heterotrophic lifestyle. Biol Chem 382: 1521-1539.

Maynard Smith J, Szathmáry E. 1995. The major transitions in evolution. W.H. Freeman, New York.

Montagna M, Sassera D, Epis S, Bazzocchi C, Vannini C, Lo N, Sacchi L, Fukatsu T, Petroni G, Bandi C. 2013. "Candidatus Midichloriaceae" fam. nov. (Rickettsiales), an ecologically widespread clade of intracellular $\alpha$-proteobacteria. Appl Environ Microbiol 79: 3241-3248.

Moreira D, Lopez-Garcia P. 1998. Symbiosis between methanogenic archaea and $\delta$-proteobacteria as the origin of eukaryotes: The syntrophic hypothesis. J Mol Evol 47: 517-530.

Müller M, Mentel M, van Hellemond JJ, Henze K, Woehle C, Gould SB, Yu RY, van der Giezen M, Tielens AG, Martin WF. 2012. Biochemistry and evolution of anaerobic energy metabolism in eukaryotes. Microbiol Mol Biol Rev 76: $444-495$.

Nakamura TM, Cech TR. 1998. Reversing time: Origin of telomerase. Cell 92: 587-590.

Neumann N, Lundin D, Poole AM. 2010. Comparative genomic evidence for a complete nuclear pore complex in the last eukaryote common ancestor. PLoS ONE 5: e13241.

Nowack EC, Melkonian M. 2010. Endosymbiotic associations within protists. Philos Trans R Soc Lond B Biol Sci 365: 699-712.

Poole A. 2010. Eukaryote evolution: The importance of the stem group. In Evolutionary genomics and systems biology (ed. Caetano-Anollés G). Wiley, Hoboken, NJ.

Poole AM, Neumann N. 2011. Reconciling an archaeal origin of eukaryotes with engulfment: A biologically plausible update of the Eocyte hypothesis. Res Microbiol 162: 71-76.

Poole A, Penny D. 2007a. Eukaryote evolution: Engulfed by speculation. Nature 447: 913.

Poole AM, Penny D. 2007b. Evaluating hypotheses for the origin of eukaryotes. Bioessays 29: 74-84.

Ramesh MA, Malik SB, Logsdon JM Jr, 2005. A phylogenomic inventory of meiotic genes; evidence for sex in Giardia and an early eukaryotic origin of meiosis. Curr Biol 15: 185-191.

Rendulic S, Jagtap P, Rosinus A, Eppinger M, Baar C, Lanz C, Keller H, Lambert C, Evans KJ, Goesmann A, et al. 2004. 
A.M. Poole and S. Gribaldo

A predator unmasked: Life cycle of Bdellovibrio bacteriovorus from a genomic perspective. Science 303: 689-692.

Rivera MC, Lake JA. 2004. The ring of life provides evidence for a genome fusion origin of eukaryotes. Nature 431: $152-155$.

Rodriguez-Ezpeleta N, Embley TM. 2012. The SAR11 group of $\alpha$-proteobacteria is not related to the origin of mitochondria. PLoS ONE 7: e30520.

Roy SW, Irimia M. 2009. Splicing in the eukaryotic ancestor: Form, function and dysfunction. Trends Ecol Evol 24: $447-455$.

Sassera D, Beninati T, Bandi C, Bouman EA, Sacchi L, Fabbi M, Lo N. 2006. "Candidatus Midichloria mitochondrii," an endosymbiont of the tick Ixodes ricinus with a unique intramitochondrial lifestyle. Int J Syst Evol Microbiol 56: 2535-2540.

Sober E. 1999. Instrumentalism revisited. Critica 31: 3-38.

Stolp H, Starr MP. 1963. Bdellovibrio bacteriovorus Gen. Et Sp. N., a predatory, ectoparasitic, and bacteriolytic microorganism. Antonie Van Leeuwenhoek 29: 217-248.

Thrash JC, Boyd A, Huggett MJ, Grote J, Carini P, Yoder RJ, Robbertse B, Spatafora JW, Rappe MS, Giovannoni SJ. 2011. Phylogenomic evidence for a common ancestor of mitochondria and the SAR11 clade. Sci Rep 1: 13.

Tong J, Dolezal P, Selkrig J, Crawford S, Simpson AGB, Noinaj N, Buchanan SK, Gabriel K, Lithgow T. 2011. Ancestral and derived protein import pathways in the mito- chondrion of Reclinomonas americana. Mol Biol Evol 28: $1581-1591$.

van der Giezen M. 2009. Hydrogenosomes and mitosomes: Conservation and evolution of functions. J Eukaryot Microbiol 56: 221-231.

van der Giezen M, Tovar J. 2005. Degenerate mitochondria. EMBO Rep 6: 525-530.

Viklund J, Ettema TJ, Andersson SG. 2012. Independent genome reduction and phylogenetic reclassification of the oceanic SAR11 clade. Mol Biol Evol 29: 599-615.

von Dohlen CD, Kohler S, Alsop ST, McManus WR. 2001. Mealybug $\beta$-proteobacterial endosymbionts contain gamma-proteobacterial symbionts. Nature 412: $433-$ 436.

Wideman JG, Gawryluk RM, Gray MW, Dacks JB. 2013. The ancient and widespread nature of the ER-mitochondria encounter structure. Mol Biol Evol 30: 2044-2049.

Williams TA, Foster PG, Nye TM, Cox CJ, Embley TM. 2012. A congruent phylogenomic signal places eukaryotes within the Archaea. Proc Biol Sci 279: 4870-4879.

Woese CR, Kandler O, Wheelis ML. 1990. Towards a natural system of organisms: Proposal for the domains $\mathrm{Ar}-$ chaea, Bacteria, and Eucarya. Proc Natl Acad Sci 87: 4576-4579.

Yutin N, Wolf MY, Wolf YI, Koonin EV. 2009. The origins of phagocytosis and eukaryogenesis. Biol Direct 4: 9. 


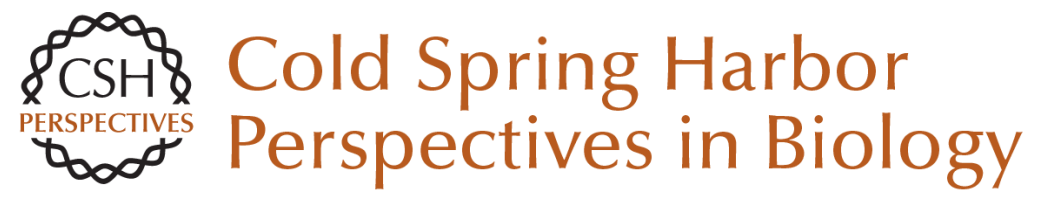

\section{Eukaryotic Origins: How and When Was the Mitochondrion Acquired?}

Anthony M. Poole and Simonetta Gribaldo

Cold Spring Harb Perspect Biol 2014; doi: 10.1101/cshperspect.a015990 originally published online July 18, 2014

\section{Subject Collection The Origin and Evolution of Eukaryotes}

The Persistent Contributions of RNA to Eukaryotic Gen(om)e Architecture and Cellular Function Jürgen Brosius

Green Algae and the Origins of Multicellularity in the Plant Kingdom James G. Umen

The Archaeal Legacy of Eukaryotes: A

Phylogenomic Perspective Lionel Guy, Jimmy H. Saw and Thijs J.G. Ettema

Origin and Evolution of the Self-Organizing Cytoskeleton in the Network of Eukaryotic Organelles Gáspár Jékely

On the Age of Eukaryotes: Evaluating Evidence from Fossils and Molecular Clocks

Laura Eme, Susan C. Sharpe, Matthew W. Brown, et al.

Origin of Spliceosomal Introns and Alternative Splicing

Manuel Irimia and Scott William Roy
Eukaryotic Origins: How and When Was the Mitochondrion Acquired?

Anthony M. Poole and Simonetta Gribaldo

Bacterial Influences on Animal Origins Rosanna A. Alegado and Nicole King

Missing Pieces of an Ancient Puzzle: Evolution of the Eukaryotic Membrane-Trafficking System Alexander Schlacht, Emily K. Herman, Mary J. Klute, et al.

The Neomuran Revolution and Phagotrophic Origin of Eukaryotes and Cilia in the Light of Intracellular Coevolution and a Revised Tree of Life

Thomas Cavalier-Smith

Protein Targeting and Transport as a Necessary Consequence of Increased Cellular Complexity Maik S. Sommer and Enrico Schleiff

How Natural a Kind Is "Eukaryote?" W. Ford Doolittle

For additional articles in this collection, see http://cshperspectives.cshlp.org/cgi/collection/

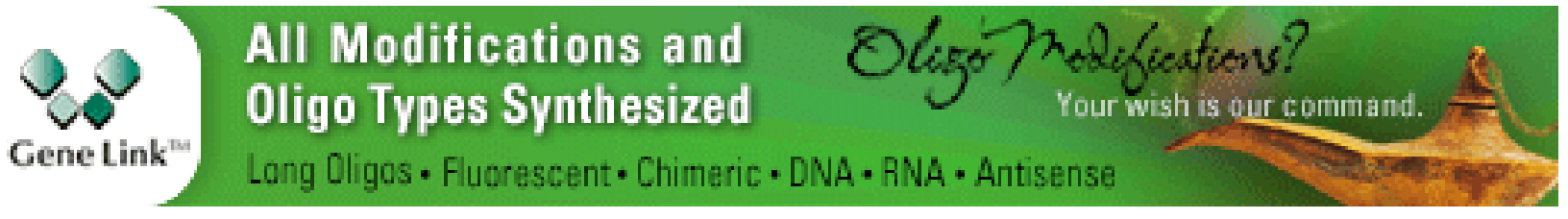


Protein and DNA Modifications: Evolutionary Imprints of Bacterial Biochemical Diversification and Geochemistry on the Provenance of Eukaryotic Epigenetics

L. Aravind, A. Maxwell Burroughs, Dapeng Zhang, et al.

The Eukaryotic Tree of Life from a Global Phylogenomic Perspective Fabien Burki
What Was the Real Contribution of

Endosymbionts to the Eukaryotic Nucleus?

Insights from Photosynthetic Eukaryotes David Moreira and Philippe Deschamps

Bioenergetic Constraints on the Evolution of Complex Life

Nick Lane

For additional articles in this collection, see http://cshperspectives.cshlp.org/cgi/collection/

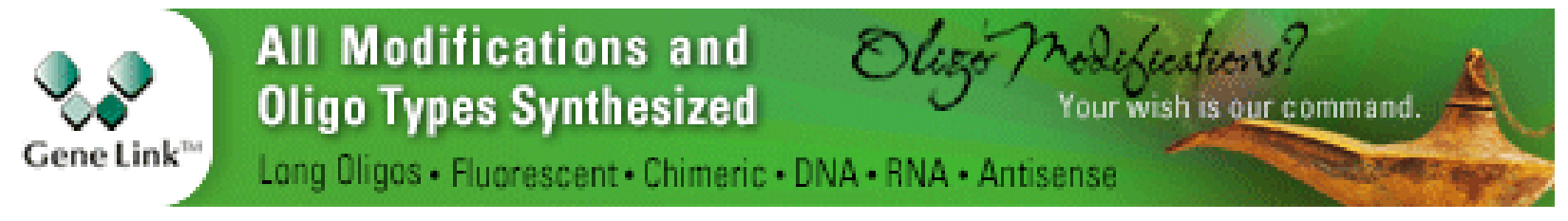

Copyright @ 2014 Cold Spring Harbor Laboratory Press; all rights reserved 